\title{
Optical Coherence Tomography Angiography of the Macula in Patients with Primary Angle-Closure Glaucoma
}

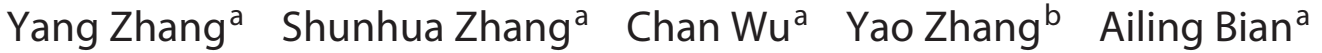 \\ Qi Zhou ${ }^{a}$ Gangwei Cheng ${ }^{a}$ Lüe Li ${ }^{a}$ \\ aDepartment of Ophthalmology, Peking Union Medical College Hospital, Peking Union Medical College, Chinese \\ Academy of Medical Sciences, Beijing, China; ' ${ }^{2}$ Clinical Epidemiology Unit, Peking Union Medical College Hospital, \\ Peking Union Medical College, Chinese Academy of Medical Sciences, Beijing, China
}

\section{Keywords}

Macular retinal vessel density . Acute primary angle closure . Optical coherence tomography angiography · Glaucoma with GCC $(p=0.017)$ and negatively correlated with LogMar BCVA $(p<0.001)$. Conclusions: After an acute angle-closure attack, the macular microvascular network is attenuated, and the MVD decreases significantly.

(c) 2020 S. Karger AG, Base

\begin{abstract}
Background: The retinal microvasculature within the macula in glaucomatous eyes is not clear. Objectives: To detect macular vessel density (MVD) changes in primary angle-closure glaucoma (PACG). Methods: Twenty-two PACG patients who had an episode of acute primary angle closure were included. Structural optical coherence tomography (OCT) scans were conducted to measure the thickness of the peripapillary retinal nerve fiber layer and macular ganglion cell complex (GCC). The MVD was measured with OCT angiography. Results: A weakened macular microvascular network that had an expanded fovea avascular zone was observed in the case group. Compared with the control group, the case group had a lower MVD $(p<0.001)$. Single correlation analysis revealed a significant correlation of the MVD with bestcorrected visual acuity (BCVA) $(r=-0.65, p=0.001), \mathrm{GCC}(r=$ $0.50, p=0.018)$, and the visual field mean deviation ( $r=$ $-0.54, p=0.009$ ) in the case group. Moreover, in the mixedeffect models, the MVD was found to be positively correlated
\end{abstract}

\section{Introduction}

As it is a type of optic neuropathy, glaucoma is characterized by both a loss of retinal ganglion cells (RGCs) and neuroretinal rim thinning of the optic nerve head $(\mathrm{ONH})$, accompanied by visual field (VF) impairment [1]. Generally, retinal nerve fiber layer (RNFL) thinning around the $\mathrm{ONH}$ is an early sign of RGC loss in glaucoma patients. It has been revealed in some studies that RGC and RNFL measurements are very accurate for diagnosing glaucoma $[2,3]$. Furthermore, there is increasing evidence that vascular insufficiency of the optic nerve is related to the onset of glaucoma $[4,5]$. According to the results of some published investigations utilizing optical coherence tomography angiography (OCTA) for quantifying the density of perfused vessels in the $\mathrm{ONH}$ and peripapillary region in glaucoma patients $[6,7]$, vessel den- 
sity measurements are strongly correlated with other glaucomatous parameters related to structure and function $[8,9]$.

Nevertheless, studies have rarely focused on the retinal microvasculature within the macular area in glaucomatous eyes. According to Chen et al. [10], measurements of the macular superficial vessel density may have the same level of diagnostic accuracy as the peripapillary RNFL and macular ganglion cell complex (GCC) thickness in differentiating primary open-angle glaucomatous and healthy eyes.

In previous studies, we have reported that peripapillary retinal vessel density is lower following an acute primary angle-closure (APAC) attack, which is considered a common cause of blindness in East Asia [11]. Subsequently, the purpose of this study was to investigate macular perfusion with OCTA. Additionally, we aimed to determine whether there is a relation between macular vessel density (MVD) measurements and other neural structures and VF loss in primary angle-closure glaucoma (PACG) patients after APAC.

\section{Materials and Methods}

The study was performed in accordance with the Declaration of Helsinki and approved by the Institutional Review Board of Peking Union Medical College Hospital. After the nature of this study was explained, every participant signed written informed consent forms prior to the start of the study.

Twenty-two consecutive Chinese patients with a history of unilateral acute PACG, who visited the Department of Ophthalmology of Peking Union Medical College Hospital between April and September 2016, were recruited for this study. The inclusion and exclusion criteria described in an earlier study were used [11]. The eyes of 22 PACG patients after APAC were included in the case group, whereas the other 22 unaffected, contralateral eyes were included in the control group. In the case group, 2 eyes were being treated with 1 local intraocular pressure (IOP) lowering medication, 1 eye was being treated with travoprost one time per day at bedtime, and another eye was being treated with brinzolamide thrice per day. Twelve eyes underwent cataract extraction, among which 5 had previously undergone a trabeculectomy. Among the unaffected eyes, no subjects needed glaucoma filtration surgery or IOP-lowering medication, and 4 eyes underwent phacoemulsification surgery. Each participant, including a total of 22 eyes across both groups, underwent laser peripheral iridotomy.

For each participant, an ophthalmologist conducted an ophthalmic examination, which included a best-corrected visual acuity (BCVA) assessment, an IOP assessment with Goldmann applanation tonometry, a slit-lamp examination, a fundus examination, gonioscopy, and a VF examination (Octopus 101 perimeter; HaagStreit Inc., Koeniz, Switzerland). MD scores can be used to assess the severity of VF loss. Spectral domain OCT (Optovue Inc., Fremont, CA, USA) was utilized for the assessment of circumpapillary RNFL (cpRNFL), GCC thickness, cup-to-disc (C/D) area ratio, and MVD.

Retinal Microvasculature within the Macular Area in PACG
Every participant underwent retina angiography examinations performed by a well-experienced ocular specialist. The scan sizes were $4.5 \times 4.5$ and $3.0 \times 3.0 \mathrm{~mm}$ for the optic disc and macular area, respectively. En face grayscale map and pseudocolor images were used to observe the morphology and quantity of the microvascular network in the macula, as well as the distribution of blood flow. The fovea avascular zone (FAZ) was depicted in an automatic manner; moreover, the area measurements for the images are provided hereinbelow. The physician altered any inaccuracy within the FAZ boundary manually. The MVD was defined as the percentage area occupied by the large vessels and microvasculature within the macular region. Image quality was evaluated for every OCT angiogram. Low-quality scans with signal strength indexes $<60$ were excluded from the analyses.

The Kolmogorov-Smirnov normality test was performed to evaluate the normality of the quantitative data. The BCVA was converted to the log of the minimal angle of resolution to conduct statistical analyses. BCVA data are presented as the median, and other data are expressed as the mean \pm standard deviation. With the nonparametric Wilcoxon test, the BCVA was compared, and with the paired-samples $t$ test, other parameters were compared between the 2 groups. Single correlation analysis was then performed to determine the relationships between MVD and traditional measurements of function and structure. Linear mixed model was used to assess the association between MVD and FAZ, GCC, BCVA, and RNFL with stratification based on PACG status and adjustment for age and IOP. SPSS version 20.0 software (SPSS for Windows; SPSS Inc., Chicago, IL, USA) was used for the statistical analyses. $p<0.05$ indicated a statistically significant difference.

\section{Results}

\section{Baseline Clinical Characteristics}

Seventeen female participants (77.3\%) and 5 male participants $(22.7 \%)$ aged between 54 and 83 years, with a mean age of $66.7 \pm 7.4$ years, were recruited in this study. The study evaluations were conducted between 7 days and 32 months, with a median duration of 22 months, after the APAC attacks.

Table 1 shows the median BCVA, the mean and range of the IOP, VF mean deviation (VFMD), GCC, RNFL thickness, and C/D area ratio. The most apparent differences between the 2 groups were revealed to be among these parameters, except for the IOP, possibly because of the careful administration of therapy following an APAC attack. Compared with the unaffected eyes, the contralateral eyes after APAC attack showed more severe VF losses and poorer BCVA. In addition, according to the assessment in structure, an elevated $\mathrm{C} / \mathrm{D}$ area ratio, as well as reduced GCC and RNFL thickness, was found among patients in the case group $(p<$ $0.001)$. 
Fig. 1. Macular microvascular network in the superficial retina of the eye after APAC attack (a) and the contralateral unaffected eye (b) in a patient. In the unaffected eye, a dense microvascular network was visible on OCTA. This network was visibly attenuated, with the enlargement of intercapillary spaces and the FAZ in the eyes after APAC attack. Pseudocolor images of the vessels in the superficial retina of the eye after APAC attack (c) showed a lower vessel density than did the contralateral unaffected eye (d). APAC, acute primary angle closure; OCTA, optical coherence tomography angiography; FAZ, fovea avascular zone.
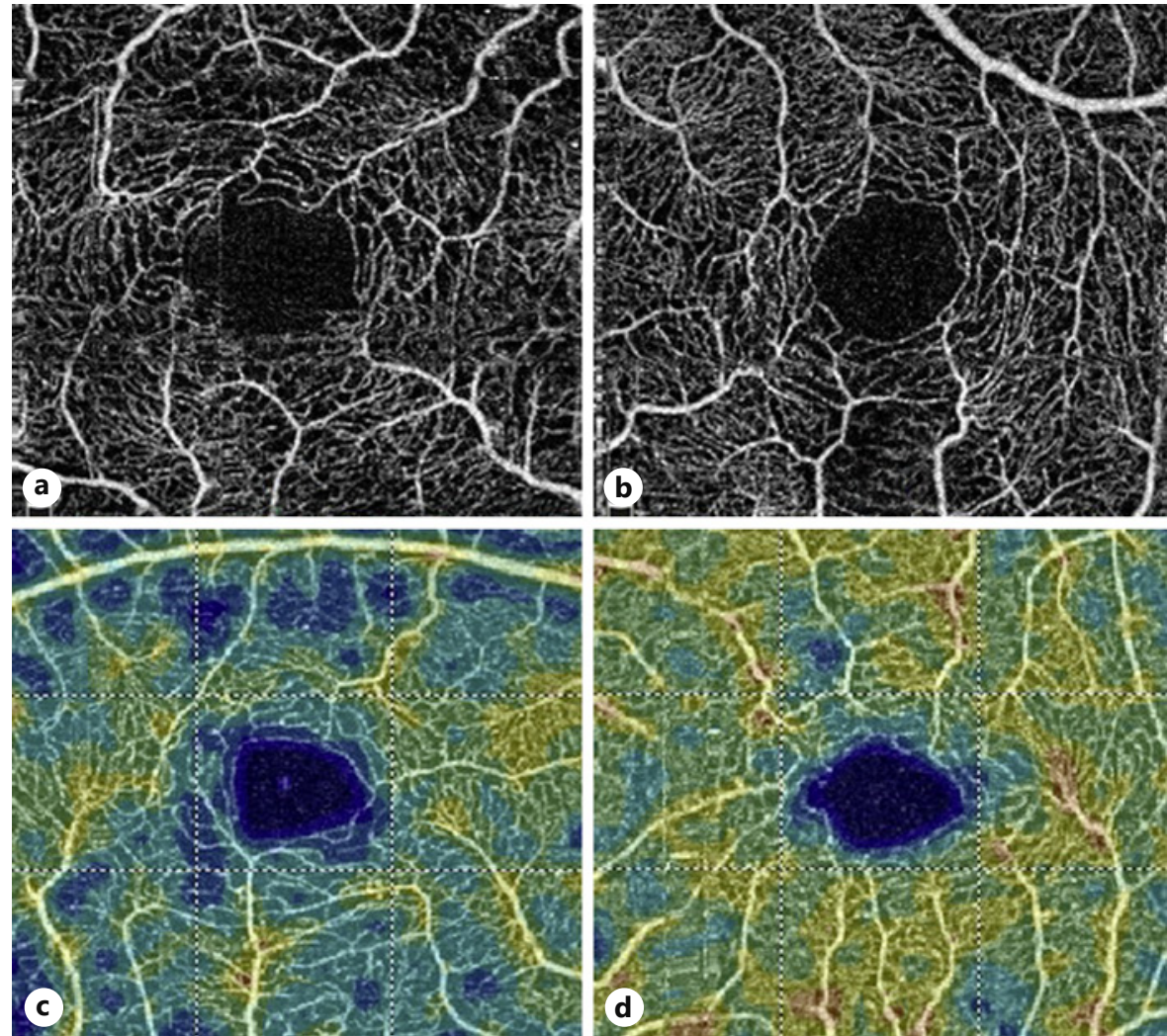

Table 1. Comparison of BCVA, IOP, VFMD, GCC thickness, RNFL thickness, and C/D area ratio between 2 groups ${ }^{\mathrm{a}}$

\begin{tabular}{|c|c|c|c|c|c|c|}
\hline \multirow[t]{2}{*}{ Variable } & \multirow{2}{*}{$\begin{array}{l}\text { Acute PACG } \\
\text { eyes }\end{array}$} & \multirow{2}{*}{$\begin{array}{l}\text { Contralateral } \\
\text { eyes }\end{array}$} & \multicolumn{3}{|c|}{ Difference } & \multirow[t]{2}{*}{$p$ value } \\
\hline & & & $\mathrm{MD}$ & LL & UL & \\
\hline BCVA, LogMar & 0.2 & 0.0 & 0.19 & 0.08 & 0.30 & 0.001 \\
\hline IOP, mm Hg & $14.66 \pm 5.32$ & $15.95 \pm 3.22$ & -1.30 & -3.56 & 0.97 & 0.101 \\
\hline VFMD, $\mathrm{dB}$ & $13.46 \pm 6.64$ & $2.57 \pm 2.36$ & 10.90 & 8.22 & 13.58 & $<0.001$ \\
\hline GCC thickness, $\mu \mathrm{m}$ & $77.6 \pm 12.8$ & $103.5 \pm 22.3$ & -25.91 & -37.63 & -14.18 & $<0.001$ \\
\hline RNFL thickness, $\mu \mathrm{m}$ & $71.5 \pm 18.5$ & $102.1 \pm 18.5$ & -30.55 & -38.94 & -22.15 & $<0.001$ \\
\hline $\mathrm{C} / \mathrm{D}$ area ratio & $0.61 \pm 0.19$ & $0.40 \pm 0.16$ & 0.21 & 0.11 & 0.31 & $<0.001$ \\
\hline
\end{tabular}

PACG, primary angle-closure glaucoma; MD, mean deviation; LL, lower limit; UL, upper limit; BCVA, best-corrected visual acuity; IOP, intraocular pressure; VFMD, visual field mean deviation IOP; GCC, ganglion cell complex; RNFL, retina nerve fiber layer. ${ }^{\text {a }} \mathrm{BCVA}$ data are presented as the median, and other data are given as mean \pm standard deviation.

\section{Comparisons among Macular Retinal Angiograms}

En face OCTA scans can provide detailed images showing either macular microvascular or vessel density from various levels. Figure 1 shows examples of superficial retina images for the 2 groups. En face OCTA scans show a denser microvascular network in the control group than in the case group, which become sparse and exhibits macular microvascular network atrophy to varying extents, enlarged intercapillary spaces, and a larger FAZ. The FAZ was not regular in terms of the contour; the perivascular space increased in size in these patients. Additionally, pseudocolor images of the vessels showed a low vessel density visually in the eyes of the case group. 
Table 2. Comparison of vessel density and FAZ area between 2 groups

\begin{tabular}{|c|c|c|c|c|c|c|}
\hline \multirow[t]{2}{*}{ Vessel density, \% } & \multirow{2}{*}{$\begin{array}{l}\text { Acute PACG } \\
\text { eyes }\end{array}$} & \multirow{2}{*}{$\begin{array}{l}\text { Contralateral } \\
\text { eyes }\end{array}$} & \multicolumn{3}{|c|}{ Difference } & \multirow[t]{2}{*}{$p$ value } \\
\hline & & & $\mathrm{MD}$ & LL & UL & \\
\hline \multicolumn{7}{|l|}{ Superficial retina } \\
\hline Whole & $45.30 \pm 3.86$ & $50.88 \pm 3.88$ & -5.58 & -7.52 & -3.64 & $<0.001$ \\
\hline Fovea & $23.72 \pm 4.07$ & $26.42 \pm 6.15$ & -2.70 & -4.76 & -0.63 & 0.013 \\
\hline Parafovea & $47.99 \pm 4.24$ & $53.28 \pm 3.67$ & -5.28 & -7.28 & -3.28 & $<0.001$ \\
\hline Superior half & $48.22 \pm 4.16$ & $52.86 \pm 4.04$ & -4.64 & -6.56 & -2.71 & $<0.001$ \\
\hline Inferior half & $47.77 \pm 4.52$ & $53.70 \pm 3.63$ & -5.93 & -8.17 & -3.69 & $<0.001$ \\
\hline Tempo & $48.19 \pm 3.86$ & $52.77 \pm 3.85$ & -4.58 & -6.78 & -2.38 & $<0.001$ \\
\hline Superior & $47.28 \pm 5.38$ & $52.76 \pm 4.56$ & -5.47 & -7.57 & -3.37 & $<0.001$ \\
\hline Nasal & $48.41 \pm 4.31$ & $52.89 \pm 3.86$ & -4.47 & -6.56 & -2.39 & $<0.001$ \\
\hline Interior & $48.09 \pm 4.94$ & $54.72 \pm 3.91$ & -6.63 & -9.21 & -4.06 & $<0.001$ \\
\hline \multicolumn{7}{|l|}{ FAZ area, $m m 2$} \\
\hline Whole & $0.43 \pm 0.13$ & $0.37 \pm 0.13$ & 0.06 & 0.03 & 0.09 & 0.001 \\
\hline
\end{tabular}

PACG, primary angle-closure glaucoma; MD, mean deviation; LL, lower limit; UL, upper limit; FAZ, fovea avascular zone.

Table 3. Correlations among the MVD and other glaucoma parameters in the case group

\begin{tabular}{|c|c|c|c|c|c|c|c|}
\hline Variables & MVD & GCC thickness & RNFL thickness & BCVA & IOP & VFMD & $\mathrm{C} / \mathrm{D}$ area ratio \\
\hline GCC thickness & $0.500(0.018)$ & & & & & & \\
\hline RNFL thickness & $0.323(0.143)$ & $0.891(<0.001)$ & & & & & \\
\hline BCVA & $0.656(0.001)$ & $0.158(0.483)$ & $0.004(0.985)$ & & & & \\
\hline IOP & $-0.399(0.066)$ & $-0.300(0.175)$ & $-0.238(0.287)$ & $-0.029(0.897)$ & & & \\
\hline VFMD & $-0.543(0.009)$ & $-0.759(<0.001)$ & $-0.749(<0.001)$ & $-0.349(0.111)$ & $0.279(0.208)$ & & \\
\hline $\mathrm{C} / \mathrm{D}$ area ratio & $-0.368(0.092)$ & $-0.694(<0.001)$ & $-0.794(<0.001)$ & $-0.031(0.891)$ & $0.410(0.058)$ & $0.667(0.001)$ & \\
\hline
\end{tabular}

MVD, macular vessel density BCVA, best-corrected visual acuity; IOP, intraocular pressure; VFMD, visual field mean deviation IOP; GCC, ganglion cell complex; RNFL, retina nerve fiber layer.

\section{Macular OCTA Measurement and Its Correlation with Regular Diagnostic Parameters}

Posterior-pole macular areas were classified into various regions automatically; moreover, the vessel density in varying areas was measured in all subjects. The vessel density values of the superficial retina were observed to be obviously lower in the case group than in the control group $(p<0.05)$. The FAZ in the case group was also apparently smaller than that in the control group (Table 2).

In the eyes of the case group, the correlation analyses revealed a significant correlation between vessel density and VFMD, BCVA, and GCC; no correlation was found with the IOP, C/D area ratio, or RNFL thickness (Table 3; Fig. 2). GCC has been found to be significantly correlated with VFMD and RNFL thickness. The MVD was not cor-

Retinal Microvasculature within the Macular Area in PACG related with the GCC, VFMD, IOP, BCVA, C/D area ratio, or RNFL thickness in the control group.

In the mixed-effect models, when controlling for age and IOP, the MVD was found to be positively correlated with GCC $(p=0.017)$ and negatively correlated with LogMar BCVA $(p<0.001)$ in the eyes of the case group (Table 4$)$. It was not correlated with these parameters in the control group.

\section{Discussion}

Currently, in clinical practice, physicians generally focus on characteristics indicating neural tissue loss in the $\mathrm{ONH}$ and its ambient areas when assessing changes in 
Table 4. Mixed-effect model analysis of MVD in the case group

\begin{tabular}{lc}
\hline Variables & MVD $^{\mathrm{a}}$ \\
\hline BCVA & $-9.88(-15.21,-4.55), p<0.001$ \\
GCC thickness & $0.11(0.05,0.16), p=0.017$ \\
RNFL thickness & $0.03(0.01,0.07), p=0.408$ \\
FAZ & $-4.81(-14.43,4.8), p=0.307$ \\
\hline
\end{tabular}

MVD, macular vessel density BCVA, best-corrected visual acuity; GCC, ganglion cell complex; RNFL, retina nerve fiber layer; FAZ, fovea avascular zone. A generalized linear mixed model was used. FAZ, GCC thickness, BCVA, and RNFL thickness were included as the fixed effect, and subject was considered as the random effect. An unstructured variance and covariance matrix was used. Ken-Roger approximation was applied to adjust the degree of freedom. A $p$ value of 0.012 was considered as statistically meaningful to ensure the family-wise error rate under 0.05 . ${ }^{\text {a }}$ Slope coefficient (95\% confidence interval), $p$ value.

the structure of glaucomatous eyes. Nevertheless, although alterations occur in the ONH and cpRNFL, the macula is suggested to be an assessment location for glaucoma [12]. Only a few large vessels exist in the macula, and the shape is normally less variable than that of the $\mathrm{ONH}$. In addition, RGCs are positioned densely inside the macula [13]. Moreover, the cell bodies of RGCs are quite large, which enables changes within the macular region to be detected easily [14]. Hence, the macular areas should be explored.

In previous studies on the macular area, the GCC thickness was the only measurement that investigators could obtain. Vessels and blood flow inside the macular area, which can indicate macular functions, could not be assessed prior to the advent of OCTA. With the wide application of OCTA in clinical practice, the superficial microvasculature in the macular and peripapillary areas in primary open-angle glaucoma has been reported [8]. For eyes possessing a history of APAC, alterations in morphological features and vessel density inside the macula have been observed, and the results are consistent with the alterations in the peripapillary retina observed by OCTA in our study [11]. Therefore, in this study, the vessel density inside the macular areas could be measured by OCTA in unilateral APAC participants. Moreover, the MVD in the eyes of the control group was identical to that found in the eyes of the unaffected participants [10], suggesting that APAC results in an abated microvascular network in the case group.

Few studies on vascular morphology and blood perfusion in PACG have been conducted. According to Gao et

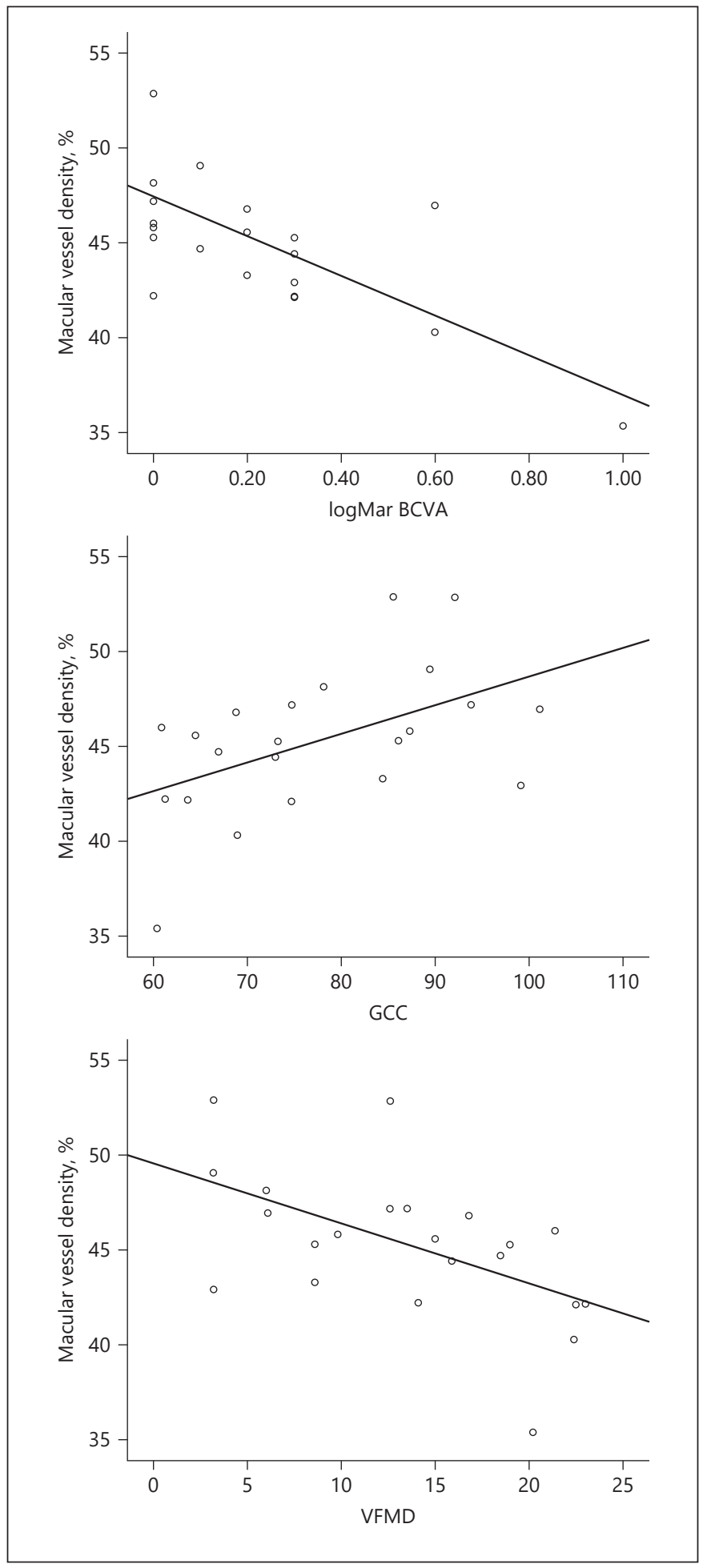

Fig. 2. Correlation of the MVD with BCVA, VFMD, and GCC in eyes of the case group. The simple linear regression results showed a negative correlation of the MVD with the VFMD and LogMar BCVA and a positive correlation with the GCC in the case group. MVD, macular vessel density; BCVA, best-corrected visual acuity; VFMD, visual field mean deviation; GCC, ganglion cell complex. 
al. [15], digital photos showed narrower retinal arteries and veins among PACG individuals, according to measurements of their retinal vessel diameter. It can be inferred that mechanical oppression likely blocks ocular blood flow temporarily; reopening and regeneration may occur in the microcirculation system after the IOP is controlled. Nevertheless, irreversible damage was found in the macular microcirculation system, which may be detected if the IOP is controlled and stabilized over multiple years. It was further demonstrated in this study that decreased blood flow was not a temporary manifestation under extremely high IOP but rather a result of extensive damage to the microvascular system.

It was revealed that MVD is more strongly connected with BCVA and GCC compared with other structural parameters, indicating an association between a sharp decrease in MVD and a more severe visual injury. The FAZ is important for good vision, and significant capillary dropout from this region is likely to lead to serious visual impairment. Therefore, macular microvascular network atrophy, decreased MVD, enlarged intercapillary spaces, and the FAZ were deemed indicators for poor visual prognoses in patients who had a history of APAC.

In a few studies, the diagnostic capabilities of macular thickness, RNFL thickness, and ONH parameters were compared. Macular measurements have been shown to offer equal levels of diagnostic abilities for glaucoma as RNFL thickness [16]. Three classes of glaucoma, differing in disease severity, were identified by Kim et al. [17] on the basis of the VFMD. The research group revealed that the macular GCC and cpRNFL thicknesses demonstrated the same diagnostic performance in the detection of early, moderate, and severe glaucoma, that macular thickness decreases due to a loss of RGCs, and that this change is correlated with either RNFL thickness or VF defects, which were previously found in glaucomatous eyes. Chen et al. [10] suggested that MVD has a higher diagnostic accuracy than do other known metrics, such as cpRNFL and GCC thickness, in differentiated primary open-angle glaucomatous and unaffected eyes. Therefore, the MVD and GCC thickness are closely correlated, suggesting the significance of MVD measured by OCTA in controlling the progression, severity, and prognosis of glaucoma. This hypothesis needs to be tested by additional studies.

Here, participants with unilateral PACG, where one eye showed symptoms of an APAC attack and glaucomatous optic neuropathy while the other eye showed no evidence of APAC, were selected as the research subjects. Self-control studies can eliminate the effects of factors such as age, sex, and systemic vascular disease, allowing conclusions to be made regarding vascular factors during the pathological process of glaucoma and increasing the reliability of the research results [18].

This research has several limitations. Primarily, a comparatively limited number of participants were enrolled. Next, more eyes in the case group than in the control group underwent cataract extraction or trabeculectomy, and there are no data about the influence of these surgeries on MVD. The time sequence of macular microcirculation abnormalities and other glaucomatous alterations was inconclusive in the present study. Then, there are still some factors that may affect MVD, such as duration of attack and peak IOP, which have not been explored in this paper. Finally, the operator performed examinations and measurements for all subjects without blinding to the study groups. Since OCTA software automatically generates reports, it can be inferred that blinding has a small effect on the measurements.

\section{Conclusion}

Until now, little data on morphological and quantitative macular perfusion in PACG patients after APAC measured with OCTA were available. In this study, macular retinal perfusion was visualized and analyzed quantitatively in a group of patients with unilateral PACG. The MVD, BCVA, and GCC thickness were observed to be strongly linked. OCTA has enabled quantitative analyses of optical and retinal flow to be conducted. There is a large amount of interest in using macular perfusion, as measured with OCTA, as a prognostic indicator for the disease course, and follow-ups of glaucomatous eyes using OCTA may improve glaucoma detection.

\section{Statement of Ethics}

This study was approved by the Institutional Review Board of Peking Union Medical College Hospital and conformed to the tenets of the Declaration of Helsinki. Written informed consent was obtained from all subjects after an explanation of the nature of the study before entry into this study.

\section{Conflict of Interest Statement}

All authors declare no financial or competing interests or relationships. 


\section{Funding Sources}

The authors did not receive any funding.

\section{Author Contributions}

Y.Z. wrote the main manuscript text and prepared all figures. Q.Z. oversaw the project and assisted with the writing of the manuscript. A.L.B. performed ophthalmic examinations. All authors reviewed the manuscript.

\section{References}

1 Jeoung JW, Choi YJ, Park KH, Kim DM. Macular ganglion cell imaging study: glaucoma diagnostic accuracy of spectral-domain optical coherence tomography. Invest Ophthalmol Vis Sci. 2013;54(7):4422-9.

2 Kim HJ, Lee SY, Park KH, Kim DM, Jeoung JW. Glaucoma diagnostic ability of layer-bylayer segmented ganglion cell complex by spectral-domain optical coherence tomography. Invest Ophthalmol Vis Sci. 2016;57(11): 4799-805.

3 Garas A, Vargha P, Holló G. Diagnostic accuracy of nerve fibre layer, macular thickness and optic disc measurements made with the RTVue-100 optical coherence tomograph to detect glaucoma. Eye. 2011;25(1):57-65.

4 Yarmohammadi A, Zangwill LM, Diniz-Filho A, Suh MH, Yousefi S, Saunders LJ, et al. Relationship between optical coherence tomography angiography vessel density and severity of visual field loss in glaucoma. Ophthalmology. 2016;123(12):2498-508.

5 Tobe LA, Harris A, Hussain RM, Eckert G, Huck A, Park J, et al. The role of retrobulbar and retinal circulation on optic nerve head and retinal nerve fibre layer structure in patients with open-angle glaucoma over an 18-month period. Br J Ophthalmol. 2015; 99(5):609-12.

6 Scripsema NK, Garcia PM, Bavier RD, Chui TY, Krawitz BD, Mo S, et al. Optical coherence tomography angiography analysis of perfused peripapillary capillaries in primary open-angle glaucoma and normal-tension glaucoma. Invest Ophthalmol Vis Sci. 2016; 57(9):OCT611-20.

7 Akagi T, Iida Y, Nakanishi H, Terada N, Morooka S, Yamada H, et al. Microvascular density in glaucomatous eyes with hemifield visual field defects: an optical coherence tomography angiography study. Am J Ophthalmol. 2016; 168:237-49.

8 Liu L, Jia Y, Takusagawa HL, Pechauer AD, Edmunds B, Lombardi L, et al. Optical coherence tomography angiography of the peripapillary retina in glaucoma. JAMA Ophthalmol. 2015;133(9):1045-52.

9 Lee EJ, Lee KM, Lee SH, Kim TW. OCT angiography of the peripapillary retina in primary open-angle glaucoma. Invest Ophthalmol Vis Sci. 2016;57(14):6265-70.

10 Chen HS, Liu CH, Wu WC, Tseng HJ, Lee YS. Optical coherence tomography angiography of the superficial microvasculature in the macular and peripapillary areas in glaucomatous and healthy eyes. Invest Ophthalmol Vis Sci. 2017;58(9):3637-45.

11 Zhang S, Wu C, Liu L, Jia Y, Zhang Y, Zhang $\mathrm{Y}$, et al. Optical coherence tomography angiography of the peripapillary retina in primary angle-closure glaucoma. Am J Ophthalmol. 2017;182:194-200

12 Zeimer R, Asrani S, Zou S, Quigley H, Jampel $\mathrm{H}$. Quantitative detection of glaucomatous damage at the posterior pole by retinal thickness mapping. Apilot study. Ophthalmology. 1998;105(2):224-31.

13 Curcio CA, Allen KA. Topography of ganglion cells in human retina. J Comp Neurol. 1990;300(1):5-25.

14 Tan O, Li G, Lu AT, Varma R, Huang D; Advanced Imaging for Glaucoma Study Group. Mapping of macular substructures with optical coherence tomography for glaucoma diagnosis. Ophthalmology. 2008;115(6):94956.

15 Gao J, Liang Y, Wang F, Shen R, Wong T, Peng $\mathrm{Y}$, et al. Retinal vessels change in primary angle-closure glaucoma: the Handan Eye Study. Sci Rep. 2015;5:9585.

16 Seong M, Sung KR, Choi EH, Kang SY, Cho JW, Um TW, et al. Macular and peripapillary retinal nerve fiber layer measurements by spectral domain optical coherence tomography in normal-tension glaucoma. Invest Ophthalmol Vis Sci. 2010;51(3):1446-52.

17 Kim NR, Lee ES, Seong GJ, Kim JH, An HG Kim CY. Structure-function relationship and diagnostic value of macular ganglion cell complex measurement using Fourier-domain OCT in glaucoma. Invest Ophthalmol Vis Sci. 2010;51(9):4646-51.

18 Yanagi M, Kawasaki R, Wang JJ, Wong TY, Crowston J, Kiuchi Y. Vascular risk factors in glaucoma: a review. Clin Experiment Ophthalmol. 2011;39(3):252-8. 